\title{
Arthrographic study of the rheumatoid knee. Part 1. Synovial proliferation
}

\author{
KYOSUKE FUJIKAWA
}

From the Department of Orthopaedic Surgery, School of Medicine, Keio University, 35 Shinano-machi, Shinjuku-ku, Tokyo, Japan

SUMMARY The improved method of double-contrast arthrography for the knee joint, which can give extensive information on the intra-articular components, was undertaken in 131 knee joints with classical rheumatoid arthritis. Synovial proliferation was classified by its localisation into 6 types: nonproliferated (NP); localised, subdivided into suprapatellar pouch (SPP), proper articular (PA), and posterior (POST); panarticular (PAN); and burned out type (BO). These types are intimately related to the radiological stage and pathological changes of the articular cartilage and menisci. By following the dynamic changes of synovial proliferation by arthrography the clinical course of the rheumatoid knee joint may be predicted. While in the NP and SPP types destruction of the joint is minimal, it is relatively rapid and severe in the PA and PAN types. Thus the proliferation in the joint proper has a stronger influence on joint destruction than does the suprapatellar pouch. From these results synovectomy to resect proliferated synovial tissues of the joint proper completely, and to resect those of the suprapatellar pouch only superficially in the early stage, was undertaken in 21 rheumatoid arthritic joints, giving excellent results in $80.9 \%$. The advantages of this method are discussed.

In rheumatoid arthritis, as the pathogical conditions of the knee joint are usually judged from clinical manifestations and plain radiographs only, it is difficult to understand sufficiently the changes in the intra-articular components such as the articular cartilages, menisci, ligaments, synovial tissues, and capsule. The method of the double-contrast arthrography $^{1}$ has been improved ${ }^{2}$ so that anyone can perform it without difficulty, demonstrate the changes in the soft tissues more exactly, and gain extensive and more direct intra-articular information about the rheumatoid knee joints.

In this paper the pathological conditions of the rheumatoid knee joints are discussed from the viewpoint of synovial proliferation as seen by the improved arthrographic technique.

\section{Materials and methods}

This arthrographic study was undertaken in 131 knee joints with classical rheumatoid arthritis according to

Accepted for publication 20 August 1980

Correspondence to Dr K. Fujikawa, Rheumatism Research Unit, School of Medicine, 36 Clarendon Road, Leeds LS2 9PJ. the American Rheumatism Association criteria; 34 joints were in male patients and 97 joints in female patients. The age distribution is shown in Fig. 1. The rheumatoid knee joints in this study were divided into 3 groups radiologically. ${ }^{3}$ Stage 1 comprised 56 joints (male 18, female 38 ), stage 2,53 joints (male 15 , female 38), and stage 3, 22 joints (male 1, female 21).

About 1000 arthrograms of normal knee joints aged from 8 months to 81 years old were used as a control group to compare with the pathological joints. $^{4}$

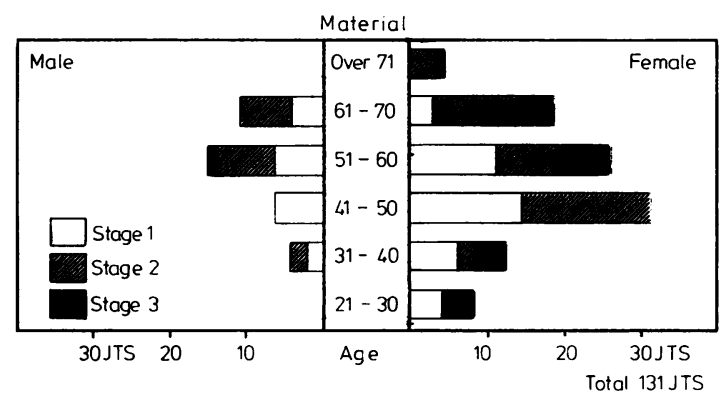

Fig. 1 Material. 
TECHNIQUE OF ARTHROGRAPHY

Sterile gloves, $5 \mathrm{ml}, 10 \mathrm{ml}$, and $50 \mathrm{ml}$ syringes, some local anaesthetic, contrast medium, a small table, and a band for fixation were required for the arthrographic procedure. Usually $70 \%$ Urografine (sodium and meglumine diatrizoates) or $60 \%$ Conray (sodium iothalamate) were used as the contrast medium. $3 \cdot 5-4 \mathrm{ml}$ of the medium, which is a suitable dose for adults, was injected into the joint cavity, followed by $40-50 \mathrm{ml}$ of air or $\mathrm{CO}_{2}$ gas. It is important to aspirate the joint fluid completely before the injection.

The joint cavity was then almost filled and expanded. The volume of air or gas was controlled

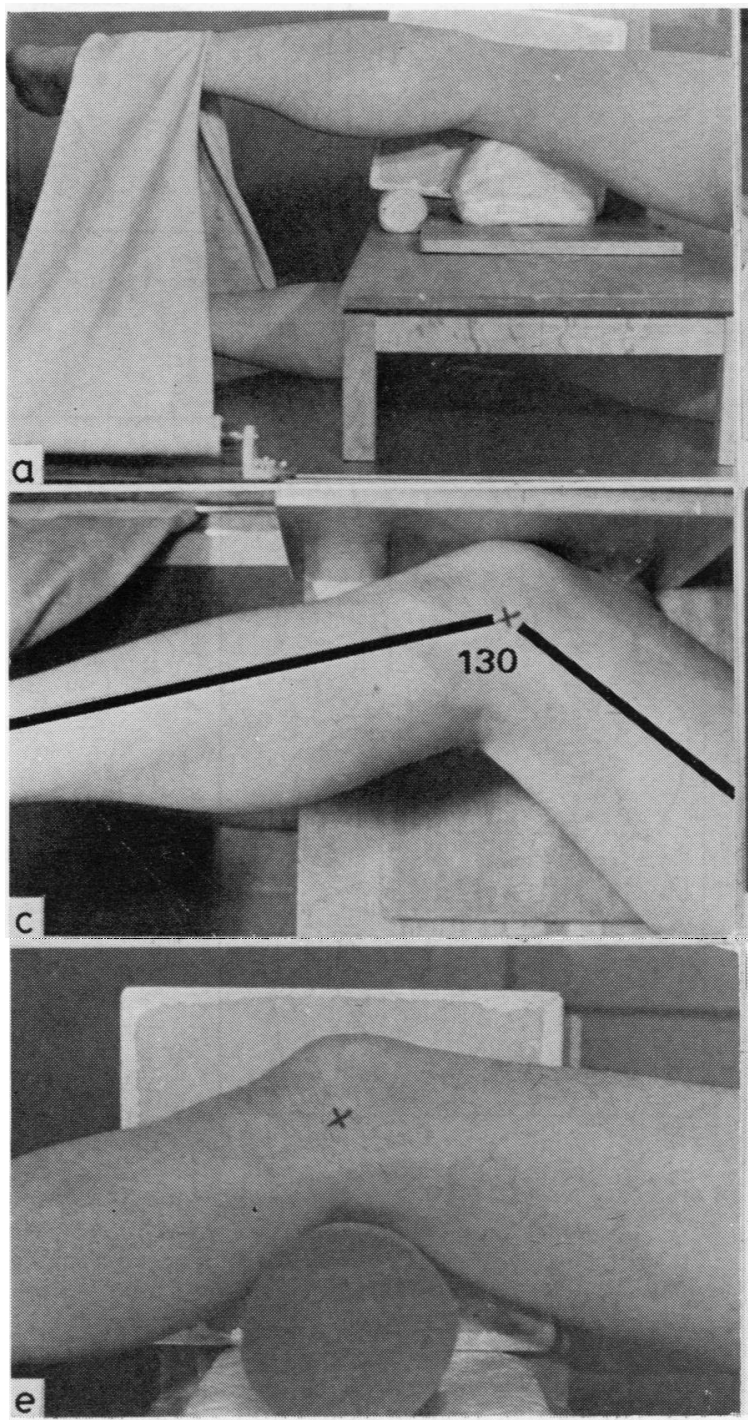

carefully, especially in the case of small females, by digital palpation of tension within the joint to prevent leakage into the extra-articular spaces, which severely inhibits accurate diagnosis. Excess dose of the contrast medium covers not only the suprapatellar pouch but also the tibio-femoral area, which makes it difficult to obtain clear arthrograms. ${ }^{5}$

After injection of the contrast medium and air the knee was rotated gently so that the medium spread over the inner surface of the joint. The patient lay down on the $x$-ray table in the lateral position with the joint space to be examined turned upwards (Fig. 2). In order to achieve clear figures of the intra-articular components such as the articular

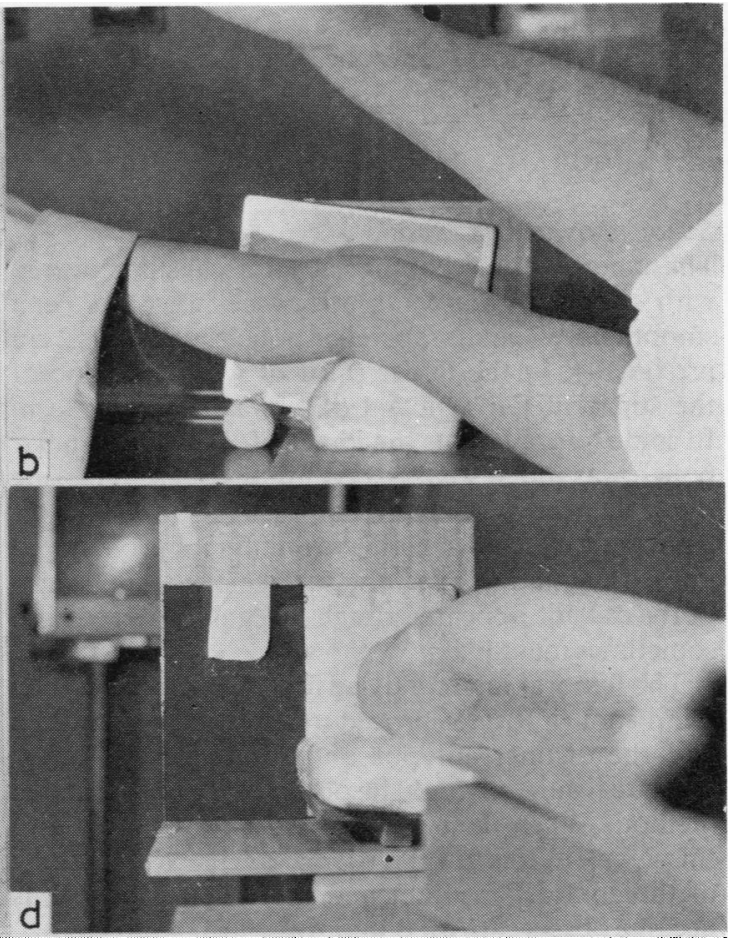

Fig. 2 Positioning in arthrographic procedure. (a) Lateral tibio-femoral compartment of the left knee (AP). (b) Medial tibio-femoral compartment of the right knee $(A P)$. (c) Flexion-angle of the knee for tibio-femoral compartment. (d) Axial view for patello-femoral compartment. (e) Lateral view (lateral arthrogram should be taken in supine position). Four arthrograms (2 AP, lateral, and axial) are routinely taken for one joint. 
cartilages, menisci, ligaments, synovial tissues and capsule it is important to spread the joint space as widely as possible, to flex the knee joint sufficiently, and to aim the $x$-ray beam parallel to the tibial articular surface. Therefore the axis of the tibia was set at $10^{\circ}$ flexion and axis of the femur at $40^{\circ}$ flexion to the $x$-ray film cassette with the patella facing it accurately. In this way the posterior angle of the knee joint becane almost $130^{\circ}$, and the joint space was opened to its maximum.

Tibial flexion to $10^{\circ}$ was necessary so that the proximal articular surface had a posterior inclination at $10-13^{\circ}$, confirmed by measurement in 250 normal adult knee joints. In this position the $x$-ray beam passed parallel to the articular surface. The femoral axis was flexed to $40^{\circ}$ because at this angle the intercondylar fossa and the joint space are most clearly visible.

\section{ARTHROGRAMS}

\section{Synovial tissue and capsule}

The inner wall of the normal joint capsule covered with the synovial membrane was shown to be a smooth line. The suprapatellar pouch spread $8-12 \mathrm{~cm}$ proximally, in the upper third of which the line of the suprapatellar synovial plica was normally seen. In some normal joints the image of the suprapatellar pouch was much smaller because the septum closing the pouch prevented the contrast medium and air from entering. When the synovial tissues proliferated in the rheumatoid knee, the surface of the inner wall of the capsule appeared rough, irregular, rugged, and sometimes mass-like.

The femoral intercondylar fossa, which has been a blind spot in former procedures, was clearly imaged and the distinct anterior and sometimes posterior cruciate ligaments could be observed by this modified method. When the synovial tissues proliferated into this area, it was displayed massively with pooling of the contrast medium and the cruciate ligaments became obscure. (Figs 3 and 4).

The menisci

The cross section of the corpus of the meniscus was imaged as an acute-angled triangle pointing its apex toward the centre of the joint. Some thin prolongations of the anterior and posterior horns spread to the tibial spines. At the upper and the lower capsular attachments of the lateral meniscus some pockets could be recognised in most cases. On the other hand, as the medial meniscus was tightly attached to the capsule, there was no space at all around the meniscus.

When the synovial tissues proliferated around the menisci, they were buried in the cloudy shadows of

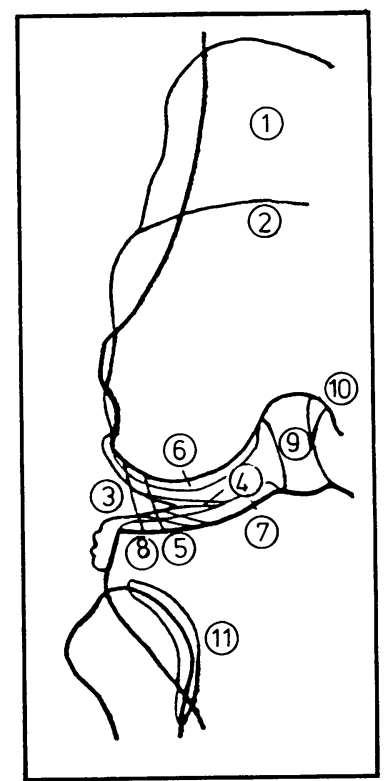

(1) A-P view (Lateral compartment)

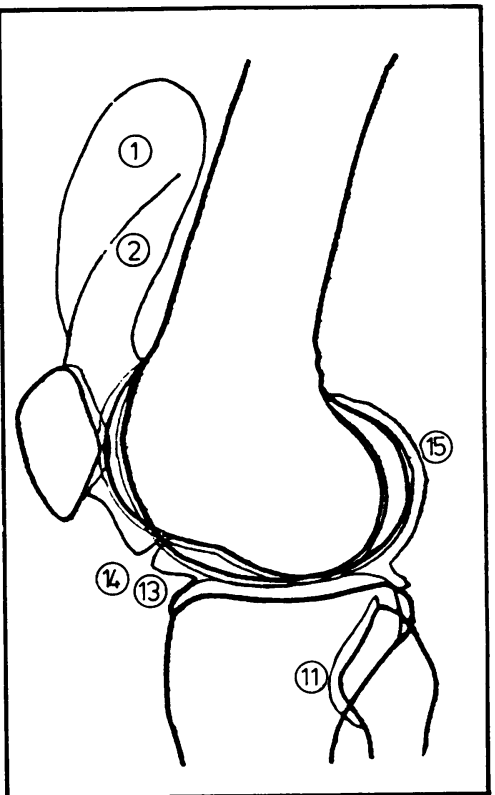

(3) Lateral veiw

Fig. 3 Schema of arthrograms of normal knee joint. (1) Suprapatellar pouch. (2) Suprapatellar synovial pleat. (3) Lateral meniscus. (4) Posterior horn of lateral meniscus. (5) Anterior horn of medial meniscus. (6) Articular cartilage (femoral condyle). (7) Articular cartilage (tibial plateau). (8) Popliteal tendon. (9) Anterior cruciate ligament.

(10) Posterior cruciate ligament. (11) Proximal tibiofibular joint. (12) Medial meniscus. (13) Infrapatellar fat pad.

(14) Infrapatellar synovial pleat. (15) Posterior capsule. 


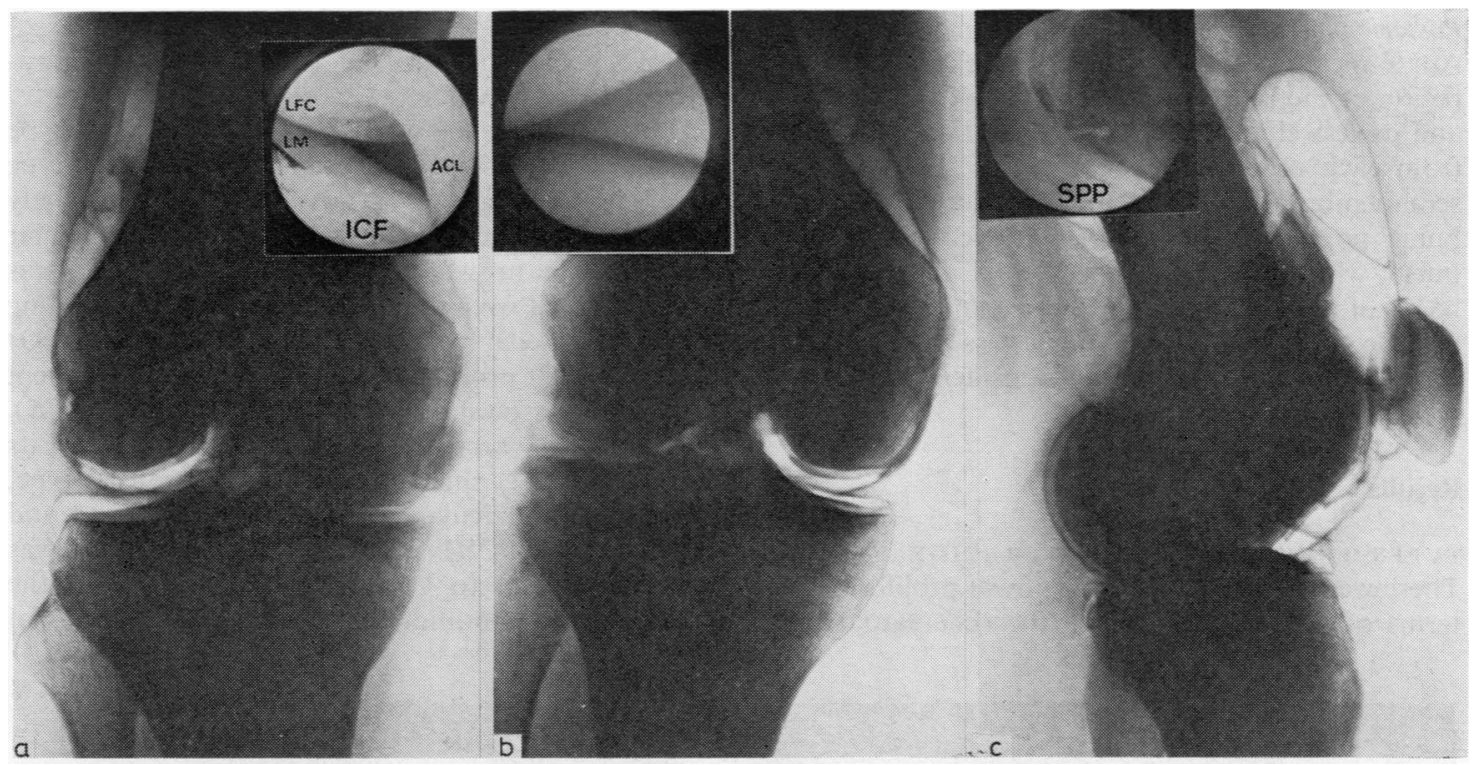

Fig. 4 Normal arthrograms (a) A-P view (lateral compartment). (b) A-P view (medial compartment). (c) Lateral view.

the pathological tissue and were invisible on the arthrograms. The outlines of the degenerative menisci with increasing deposits of the contrast medium were unclear. The severely degenerated or torn menisci also lost their original outlines by invasion of the contrast medium into the breaks and fissures.

\section{Articular cartilage}

The normal articular cartilage was displayed smoothly and evenly, and stains made by the contrast medium were thin and constant. But the complexity of the proximal tibial articular surface, of which the medial is concave and the lateral is convex, was noticed in reading the arthrograms. On the other hand the femoral articular surface was imaged clearly because the femoral configuration is globular and the $x$-ray beam struck at a tangent.

The thickness of the articular cartilage at the P-point (the apices of the medial and the lateral femoral condyles) and their opposite points on the tibial articular cartilage decreased rapidly until 15 years old, but after that it was constant at $2-3 \mathrm{~mm}$ till 80 years of age, as seen in about 1000 arthrograms of normal knee joints aged from 8 months to 81 years old. ${ }^{4}$

When the articular cartilage was eroded, its appearance became obscure with increasing deposit and pooling of the contrast medium. In cases of ulceration by invasion of the synovial tissues the surface appeared abnormal, with uneveness, localised thinning, and notable pooling of the contrast medium. In cases of advanced destruction filling defects reaching the subchondral area could be recognised.

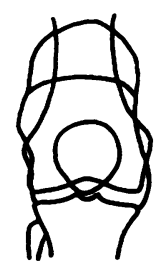

NP

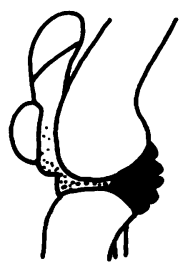

POST

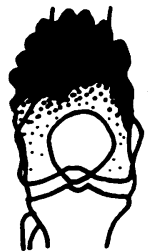

SPP

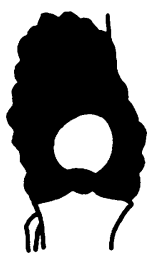

PAN

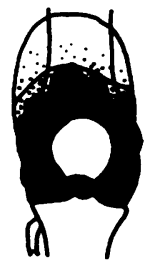

PA

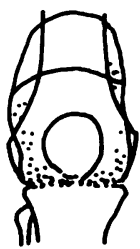

BO
Fig. 5 Type of synovial proliferation. NP:Nonproliferated type. SPP: Suprapatellar pouch localised type. PA: Proper articular localised type. POST: Posterior localised type. PAN: Panarticular type. BO: Burned out type. 


\section{Posterior bursae}

Anatomically there are 8 main bursae in the posterior region of the knee, but it was difficult radiologically and useless clinically to distinguish them accurately from each other. Usually the bursa gastrocnemiosemimembranosum was medially located and the bursa popliteum laterally. The so-called posterior bursa, which lies among the medial and the lateral heads of the gastrocnemius muscle and the posterior capsule of the joint was often missed clinically in spite of being expanded by considerable synovial proliferation in RA.

\section{Results}

TYPES OF SYNOVIAL PROLIFERATION There were various types of synovial proliferation in terms of their localisation in the rheumatoid knee.
They were closely related not only to the stages of Steinbrocker, ${ }^{3}$ but to the changes of the intraarticular components.

These types were (1) nonproliferated (NP) type without any synovial proliferation; (2) localised type in which proliferation of synovial tissue was relatively localised to some part of the joint; (3) panarticular (PAN) type in which synovial proliferation was prominent all over the intra-articular area including the suprapatellar pouch; and (4) burned out (BO) type in which proliferation had already gone except for fibrous tissue and the joint was destroyed (Fig. 5). The localised type was further subdivided by its locality into (1) suprapatellar (SPP) localised type, (2) proper articular (PA) localised type, and (3) posterior (POST) localised type (Fig. 6). It was clinically useful to know accurately not only the pathological condition but also the locality of

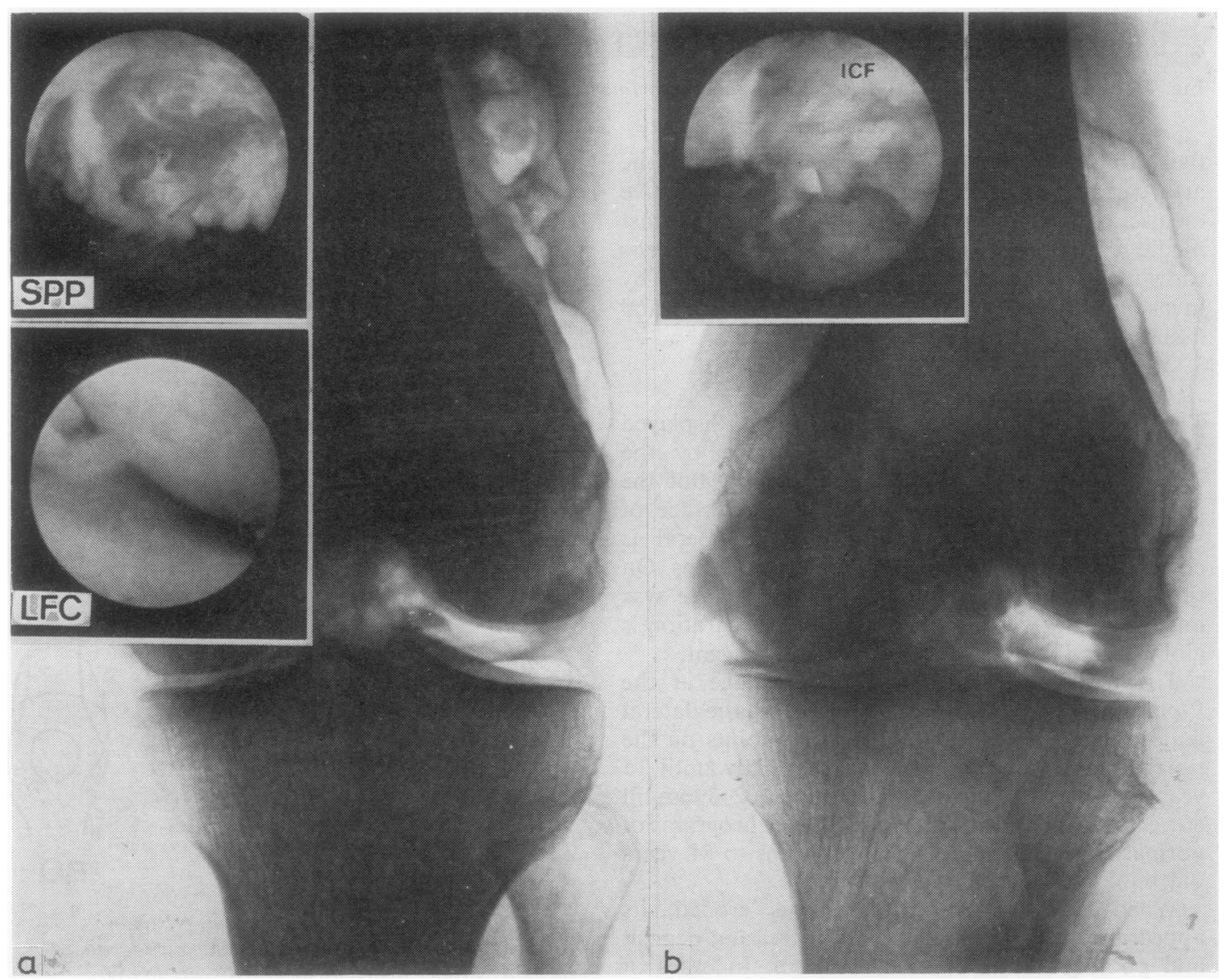

Fig. 6 (a) Suprapatellar pouch localised (SPP) type. (b) Proper articular localised (PA) type. (c) Posterior localised (POST) type. (d) Panarticular (PAN) iype. (e) Burned out (BO) type. 


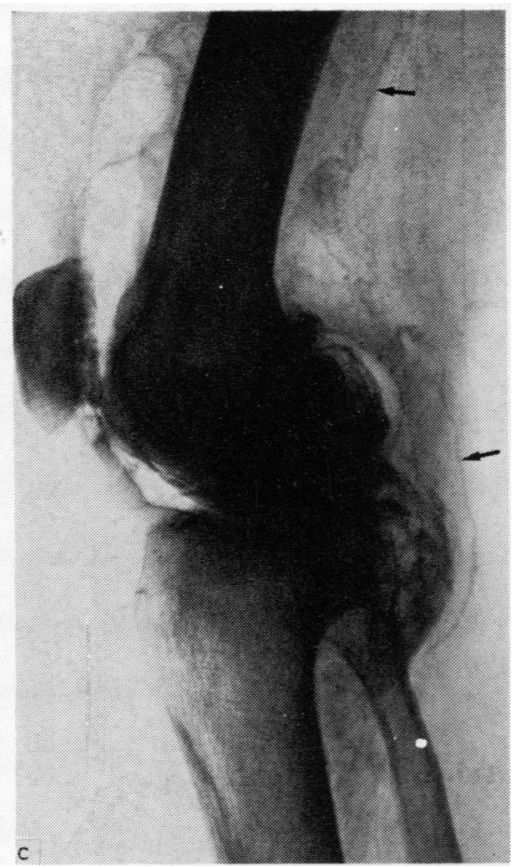

the proliferated synovial tissue, because those were the most important factors in evaluating the prognosis of the rheumatoid knee.

\section{CLINICAL MANIFESTATION AND}

ARTHROGRAPHIC FINDINGS

Swelling, which is one of the main clinical manifestations in the rheumatoid knee, is usually assessed only roughly by palpation as para-articular swelling, though it may in fact decide the prognosis of the joint.

The degree of synovial proliferation, especially in the proper articular area, namely, the PA type and the PAN type, was related to pain on movement and loading, although it was not always related to pain at rest (Fig. 7).

Hydrops was not necessarily related to the degree of synovial proliferation. Distinct synovial proliferation without hydrops was often recognised $(60 \%)$ and vice versa.

Except some advanced cases the condition of the articular cartilage and menisci had little relationship to pain at rest or even on movement or loading (Fig. 8). So the pathological conditions of those intra-articular components could not always be

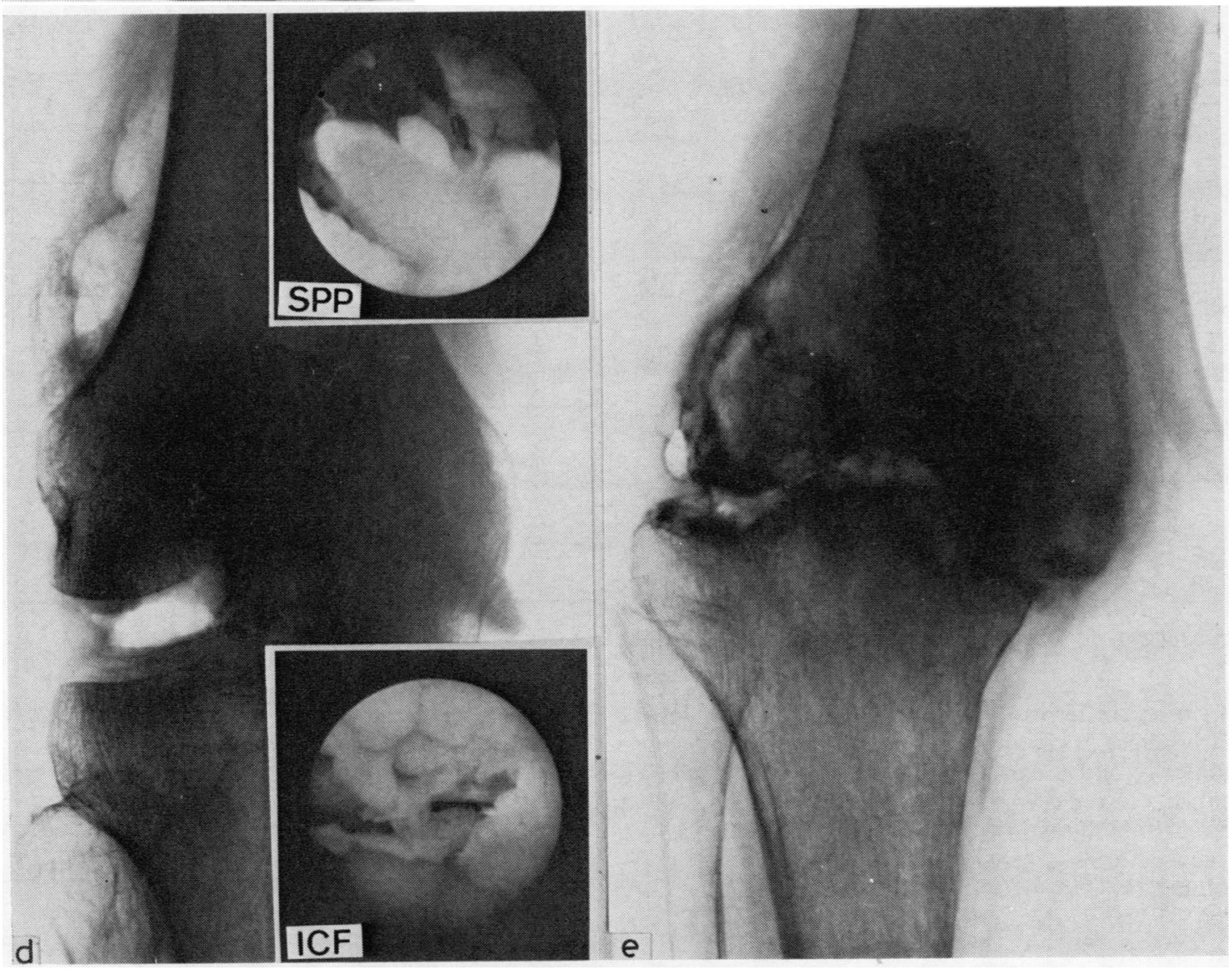




\begin{tabular}{|c|c|c|c|c|}
\hline & NP & SPP & PA \& PAN & BO \\
\hline \multicolumn{5}{|l|}{ HYDROPS } \\
\hline \multirow{2}{*}{\multicolumn{5}{|c|}{$\begin{array}{l}\text { PAIN } \\
\text { AT REST }\end{array}$}} \\
\hline & 711 & 30 & 23 & \\
\hline \multirow{2}{*}{$\begin{array}{l}\text { PAIN } \\
\text { ONLOAD }\end{array}$} & & & 22 & \\
\hline & $\square_{18}$ & & & \\
\hline & \multicolumn{2}{|c|}{ SYMPTOM(-), AG-FIND.(+) } & \multicolumn{2}{|c|}{ ]SLIGHT PROLIF } \\
\hline & \multicolumn{2}{|c|}{ SYMPTOM(t),AG-FIND.(+) } & & \\
\hline
\end{tabular}
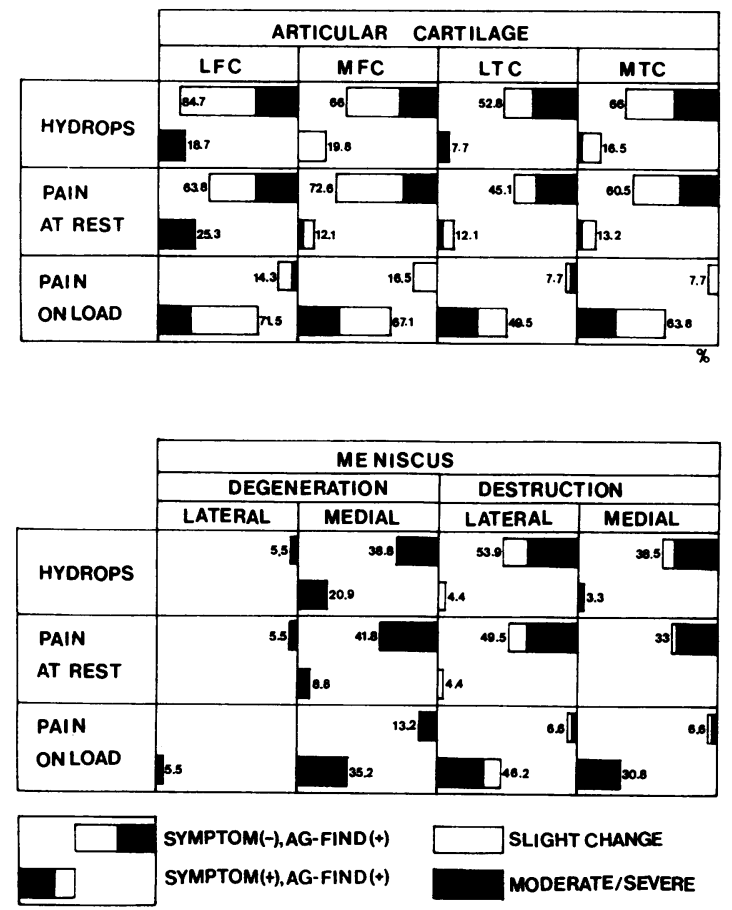

Fig. 8 Relationship between clinical symptoms and the changes of articular cartilage and menisci.

discussed in terms of clinical manifestations in the early stages. It should be noted that there were many clinically silent pathological changes of the components in the rheumatoid knee. In advanced cases, clinical manifestations such as pain, instability, and some deformity were related to destruction of the joint.
Fig. 7 Relationship between clinical symptoms and type of synovial proliferation. 
slight. In the stage 2 group the percentage of the joints with synovial proliferation increased to $82.4 \%$, in which $66.6 \%$ was the PA and PAN types. In the stage 3 group the type of synovial proliferation was virtually limited to the PA type or PAN type and the rest were of the BO type or nearly of the BO type, in which synovial proliferation had already disappeared and the joint was destroyed.

From the point of view of synovial proliferation $76.3 \%$ of the NP type were in stage 1 and the rest $(23.7 \%)$ in stage 2 . This type of proliferation was not recognised in stage 3. In the SPP type the stage 1 group accounted for $52.9 \%$ and the stage 2 for $47 \cdot 1 \%$. There was no stage 3 joint in which synovial proliferation was localised to the suprapatellar pouch. In the PA type stage 1 was $39 \cdot 1 \%$, stage 2 was $52.2 \%$, and stage 3 was $8.7 \%$. In the PAN type stage 1 was $18.4 \%$, stage 2 was $57.7 \%$, and stage 3 was $23.7 \%$. In other words, in the PA and PAN type most of the joints were in the advanced stage. All of the BO type belonged to the stage 3 group. It appears therefore that the type of synovial proliferation is intimately related to the radiological stage. The long duration of the PA and PAN type had a

\begin{tabular}{|c|c|c|c|c|}
\hline Prolif. type & No changes & Slight & Moderate & Severe \\
\hline No proliferation & UIIIA 44.7 & OIIIA 53.6 & $2 \cdot 7$ & \\
\hline Localised (SPP) & & TIIs-27II & 5.9 & \\
\hline Localised $(T-F)$ & $4 \cdot 4$ & $\mathrm{PICA}^{47.8}$ & OICa $^{39 \cdot 1}$ & 8.7 \\
\hline Pan articular & $5 \cdot 3$ & OIIAG $_{4.7}$ & $\mathscr{M}^{18.1}$ & $\operatorname{Pat}^{31 \cdot 6}$ \\
\hline Burned out & & & & S \\
\hline
\end{tabular}

Fig. 10 Relationship between type of synovial proliferation and articular cartilage. strong influence on joint destruction compared with the NP and SPP type.

\section{SYNOVIAL PROLIFERATION AND CHANGES OF THE ARTICULAR CARTILAGE}

The type of synovial proliferation and the changes of the articular cartilage were closely related (Fig. 10).

In the NP type no changes in the articular cartilage could be seen in $44.7 \%$ and only a slight change in $53.6 \%$. In the NP type $97.3 \%$ were limited to a slight change. In the SPP type $94.1 \%$ had slight changes, and in only $5.9 \%$ could moderate change in the articular cartilage be observed. In the PA type $52.2 \%$ had slight changes, moderate change was observed in $39.1 \%$, and severe change in $8.7 \%$. Furthermore, changes of the articular cartilage were much more advanced in the PAN type; severe change was seen in $36.6 \%$ of this type. In the BO type all showed severe changes. The articular cartilages were severely destroyed or had already disappeared.

It appears therefore that the changes of the articular cartilage in the NP type and the SPP type are slight, but in the PA and PAN type they are much more severe because in these types they advance rapidly. It is interesting that the types of synovial proliferation were divided into 2 groups as above and a line could be drawn between them clearly.

Even in terms of duration, especially in the stage 2 group, the articular cartilages of the PA and PAN type were far more severely damaged than those of the NP and SPP type.

\section{SYNOVIAL PROLIFERATION AND CHANGE IN THE MENISCI}

The relationship between synovial proliferation and changes in the menisci was similar to the changes in the articular cartilage (Fig. 11). The menisci were almost normal in their appearance in $66.7 \%$ of the NP type; $30 \cdot 8 \%$ had slight changes such as degeneration and only $2.5 \%$ of the menisci were torn in this type of proliferation. In proportion to synovial tissue

\begin{tabular}{|c|c|c|c|c|c|}
\hline $\begin{array}{l}\text { Meniscus } \\
\text { Prolif type }\end{array}$ & No change & Degenerat. & Tear & Disappear & Undetect. \\
\hline No proliferation & - & 30.8 & 2.5 & & \\
\hline Localised (SPP) & $29 \cdot 4$ & 58.8 & 5.9 & & $5 \cdot 9$ \\
\hline Localised $(T-F)$ & $23 \cdot 1$ & 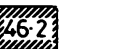 & 211.5 & $15 \cdot 4$ & 3.8 \\
\hline Pan articular & 10.8 & 58 & (16.2 & $24 \cdot 4$ & \\
\hline Burned out & & & & Ving & \\
\hline
\end{tabular}

Fig. 11 Relationship between type of synovial proliferation and meniscus. 
proliferation the changes in the menisci were advanced, but the changes in the SPP type remained slighter than those in the other types. On the other hand severe change of the menisci such as tear or disappearance were seen in $26.9 \%$ of the PA type and $40.6 \%$ of the PAN type, which indicates a relationship between the degree of synovial proliferation in the proper intra-articular space and the changes in the menisci in the rheumatoid knee. In the BO type meniscal disappearance reached almost $100 \%$.

\section{POSTERIOR REGION}

Although synovial proliferation in the posterior region of the rheumatoid knee exists in many cases, it may escape notice because of the difficulty of feeling it clinically. This proliferation was usually seen in the PAN type, sometimes in the PA type, but rarely in the SPP type. In some cases synovial proliferation was localised only in the posterior region without any relation to the anterior part of the joint, which could be classified into the posterior localised type. In relation to the stage moderate or severe proliferation made up only $22.4 \%$ in stage 1 but it increased to $68.4 \%$ in stage 2 and $100 \%$ in stage 3 .

Calf cysts or giant calf cysts were seen in $5.1 \%$ of all the joints, and these were not related to the radiological stage. The lymphatic ducts were imaged in $5.0 \%$ of the moderate and in $25 \%$ of the severe proliferated cases.

\section{CLINICAL COURSE AND SYNOVIAL}

\section{PROLIFERATION}

Seventy four knee joints were observed from 6 months to 5 years in relation to the type of synovial proliferation and the stages of joint damage (Table 1).

In the initial arthrograms 34 of 74 joints were of the NP type, 18 joints of the SPP type, 8 joints of the PA type, and 14 joints were of the PAN type. Twenty eight of 34 joints of the NP type stayed in the

Table 1 Relationship between changes of synovial proliferation type and advancement of stage by periodical arthrography.

\begin{tabular}{|c|c|c|c|}
\hline \multicolumn{2}{|c|}{$\begin{array}{l}\text { Changes of synovial proliferation } \\
\text { type }\end{array}$} & \multicolumn{2}{|c|}{$\begin{array}{l}\text { Changes of stage } \\
\text { (roentgenograms) }\end{array}$} \\
\hline Initial $A G$ & Following $A G$ & Non changes & Progressive \\
\hline $\begin{array}{l}\text { NON (34) } \\
\text { SPP (18) } \\
\text { PA (8) } \\
\text { PAN (14) } \\
\text { Total: } 74 \text { J }\end{array}$ & $\begin{array}{l}\text { NON or SPP } \\
\text { PA or PAN } \\
\text { NON or SPP } \\
\text { PA or PAN } \\
\text { NON or SPP } \\
\text { PA or PAN } \\
\text { BO } \\
\text { NON or SPP } \\
\text { PA or PAN } \\
\text { BO }\end{array}$ & $\begin{array}{c}26(92 \cdot 9) \\
3(50 \cdot 0) \\
13(100) \\
0(0) \\
1(100) \\
2(33 \cdot 3) \\
0(0) \\
2(100) \\
5(55 \cdot 5) \\
0(0)\end{array}$ & $\begin{array}{l}2(7 \cdot 1) \\
3(50 \cdot 0) \\
0(0) \\
5(100) \\
0(0) \\
4(66 \cdot 7) \\
1(100) \\
0(0) \\
4(44 \cdot 5) \\
3(0)\end{array}$ \\
\hline
\end{tabular}

same type group or changed to the SPP type, and all except 2 joints showed no advancement in the stage at later arthrograms. On the other hand 6 of the 34 joints changed to the PA or PAN type, and half of them progressed in their stage. Similarly, 13 improved to the NP type from the SPP type or stayed as the SPP type and did not show any radiological deterioration. In 8 PA types at initial arthrography there was only 1 joint which improved to the SPP type, and the radiological stage did not progress, but 6 continued to be of the PA type and one advanced to the BO type and then they deteriorated radiologically.

In 14 joints of the PAN type those which improved to the NP or SPP type stayed in the same radiological stage. Four of 9 joints which stayed as the PA or PAN type deteriorated radiologically. Three joints which advanced to the BO type showed considerable radiological deterioration.

By following the dynamic changes of synovial proliferation by means of arthrography for years the clinical course of the rheumatoid knee joint may be predicted, as shown in Fig. 12. While in the NP and the SPP types destruction of the joint is minimal, it is relatively rapid and severe in the PA and PAN type. Thus a line may be drawn between the NP, SPP type and the PA, PAN type, which seems to be the critical point for the joint destruction.

If the purpose of early synovectomy of the rheumatoid knee is to prevent or to mitigate the destruction of the intra-articular components, it may be indicated for those joints in the early stages which have continued in the PA or the PAN type so long as some initial destructive changes have appeared on the arthrograms.

The method of synovectomy which I have tried for some years is to resect the proliferated synovial tissues of the proper knee joints as completely as possible to the fibrous capsule and to resect superficially those of the suprapatellar pouch, which is the proximal area of the suprapatellar synovial plica. The advantages of this procedure are not only that operative invasion is minimal and the range of motion of the joint after surgery as mentioned by Marmor and others ${ }^{6-8}$ is unimpaired, but the

\section{SPECULATION OF CLINICAL COURSE}

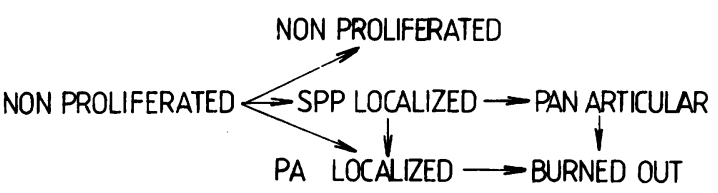

Fig. 12 Speculation of clinical course of synovial proliferation. 


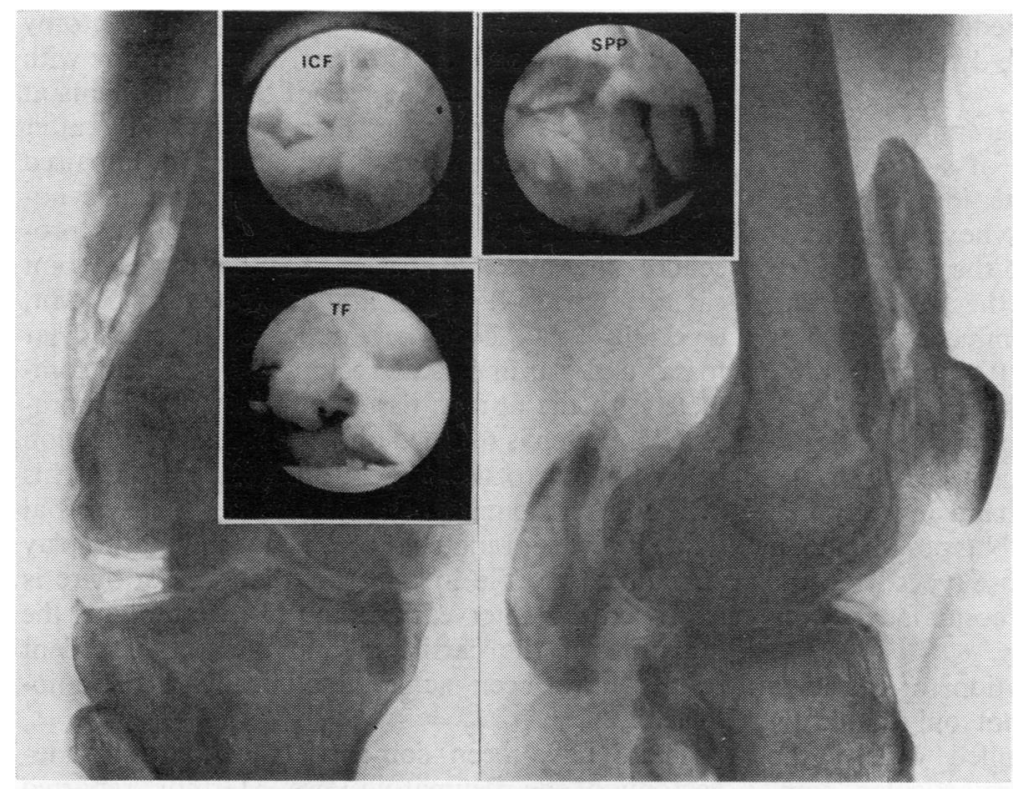

Fig. 13a Male, 32 years old.

Classical rheumatoid arthritis. Arthrograms before operation (PAN type with Baker's cyst).

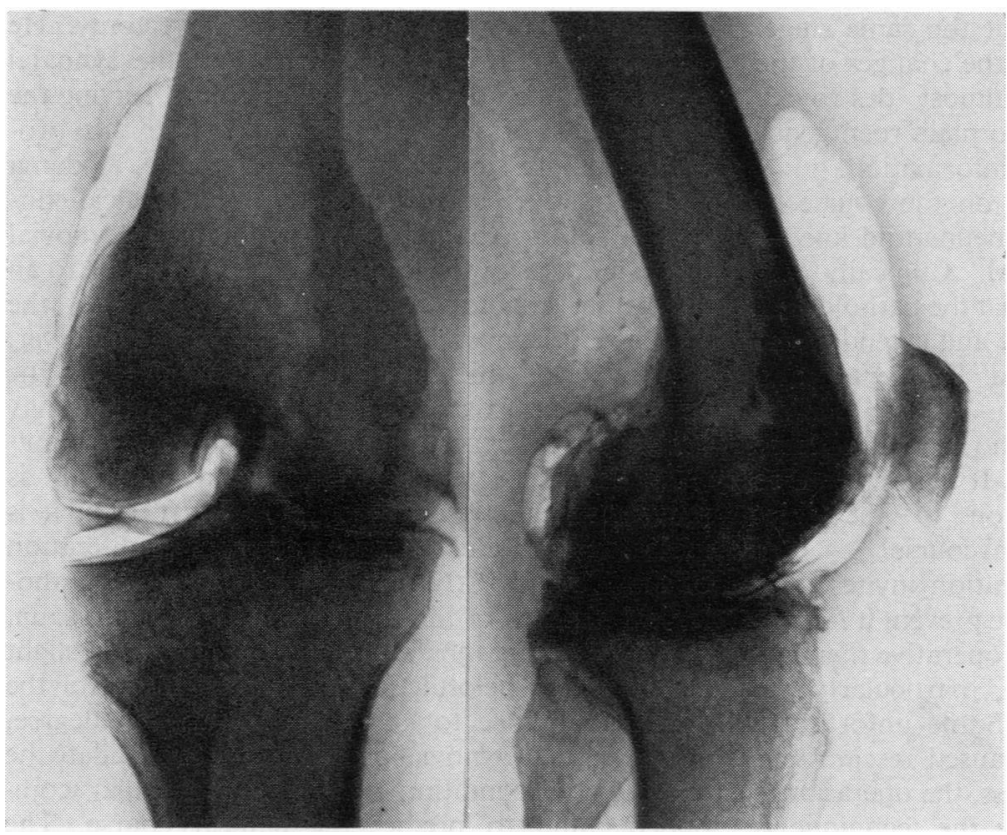

Fig. 13b Arthrograms of 5 years after operation (partial synovectomy). Synovial proliferation has completely subsided and articular cartilage and menisci were well maintained.

nutrition to the articular cartilage, by avoiding total resection of the synovium, is maintained also. Twenty one cases by this procedure were followed over 5 years (Fig. 13); the results were excellent in $80.9 \%$. They showed no advancement in the stage at all and the type of the synovial proliferation was maintained in the NP $(76.5 \%)$ or the SPP $(23.5 \%)$.

\section{Discussion}

In general, pathological changes in the rheumatoid knee joint begin from the synovial tissue and extend to the intra-articular components, such as the articular cartilage, menisci, and ligaments. There is no doubt that the change in the articular cartilage especially influences the prognosis of the joint 
function, so it is important to predict the clinical course of the joint by following the dynamic change of synovial proliferation.

By applying the improved arthrographic technique to the rheumatoid knee the state of synovial proliferation can be classified by site into the types mentioned above. The course of synovial proliferation appears to be the NP type, to the SPP type, to the PA type, to the PAN type, to the BO type. The PA and PAN type have more influence on joint destruction than do the NP and SPP type. In fact, by following the dynamic changes of synovial proliferation in 74 rheumatoid knee joints periodically for several years we found few advanced cases in the NP and SPP type. On the other hand in the joints which have been in the PA and PAN types for a long time or have been converted to those types from the former 2 types, the intra-articular components were destroyed more rapidly and severely.

Though Steinbrocker's classification ${ }^{3}$ of the stage by the plain roentgenograms is not only clinically useful but also simple and applied widely, the pathological changes of the intra-articular components vary considerably even at the same stage. Particularly in the stage 2 group, the changes of the joints vary from normal to almost destroyed. Arthrograms are much superior to plain roentgenograms in providing intra-articular information.

There are 2 different opinions on the course of cartilagenous destruction in the rheumatoid kneeextrachondral and intrachondral. Clinically the course of some patients suggests that the pathological changes begin from synovial tissue and extend to the articular cartilages, menisci and ligaments, and finally produce joint destruction. As the clinical course is not always uniform, each rheumatoid knee requires individual consideration. It is important to know the pathological condition of the joint accurately and to predict the clinical course.

What kind of synovial proliferation invites joint destruction rapidly and how can we prevent it? There is still much discussion about the operative management of the rheumatoid knee, particularly on indications for synovectomy. ${ }^{910}$ Some unfortunate results from synovectomy have caused reservations about the procedure. ${ }^{10}$ Nevertheless, the operation is still popular and useful, because the technique is simple and results are frequently excellent. ${ }^{6}$ 11-15

Synovectomy may be divided into 2 types, early and late. Recently early synovectomy has attracted attention and the results are better than those for late synovectomy. ${ }^{72-14}$ 16-22 Results of synovectomies even by the same surgeon and at the same stage often vary. One of the causes may be evaluation of the joint only by plain roentgenograms, which cannot define the details of the joint pathology.
Our experience of total capsular synovectomy shows that in some cases of early synovectomy with minimal pathological changes of the components at operation the joint space narrowed rapidly after surgery. This is thought to be due to impaired nutrition of the cartilage. Recently, partial synovectomy has been tried in my clinic. In this procedure the synovial tissues of the proper joint cavity (patello-femoral and tibio-femoral joint, especially the condylar pouches, intercondylar fossa, and parameniscal region) are resected completely, to the fibrous tissue, and that of the suprapatellar pouch is resected only superficially, resection being limited to the grossly proliferated sites. This method is recommended because (1) the synovial tissue in the suprapatellar area is not thought to play an immediate role in joint destruction, (2) there is less operative intervention, and (3) it maintains the nutrition of the cartilages and menisci. Excellent results have been achieved clinically and radiologically.

There have been some trials of partial synovectomy of the rheumatoid knee. Marmor ${ }^{8}$ reported on about 300 joints of partial synovectomy. He showed that there was no spread of the synovial tissues into the joint proper as a result of leaving the suprapatellar tissues. He seemed to use this procedure only to simplify postoperative care. Kodama and Kondo, ${ }^{23}$ considering the pathological differences of fine vascular construction of the synovial tissues in parts of the joint, discussed and came to an interesting conclusion about, the region and the depth of the synovial tissue to be resected. However, their method, which leaves the synovial tissue in the intercondylar fossa and parameniscal region, may have much influence on the destruction of the joint.

As the main purpose of early synovectomy is prevention or alleviation of joint destruction, it is indicated for joints in which synovial proliferation of the PA and PAN type is developing, the pathological changes of the components have just begun, and the radiographs still show minimum or slight changes. It is helpful for accurate treatment of the rheumatoid knee to know the localisation degree, and changes of synovial proliferation, to elucidate the pathological condition of the intra-articular components and to predict the clinical course. The arthrographic technique should be applied broadly because it is not only simple but also useful, repeatable, and provides much intra-articular information.

The author thanks Professor V. Wright for very helpful advice and Mrs B. Hill, who kindly corrected the English.

\section{References}

1 Andren L, Wehrin H. Double contrast arthrography of knee with horizontal roentgen ray beam. Acta Orthop Scand 1960; 39: 307-14. 
2 Fujikawa K, Iseki F. Arthrography of the knee joint. Clin Orthop Surg (Jpn) 1976; 11:213-30.

3 Steinbrocker $O$. Therapeutic criteria in rheumatoid arthritis. JAMA 1949; 140: 659-62.

4 Fujikawa K, Tomatsu T, Matsu K, Koike A, Tanaka Y, Iseki F. Morphological analysis of meniscus and articular cartilage in the knee joint by means of arthrogram. $J J p n$ Orthop Assoc 1978; 52: 203-15.

5 Taylor A R, Ansell B M. Arthrography of the knee before and after synovectomy for rheumatoid arthritis. $J$ Bone Joint Surg 1972; 54B: 110-5.

6 Marmor L. Synovectomy of the rheumatoid knee. Clin Orthop 1966; 44: 151-62.

7 Marmor L. Surgery of the rheumatoid knee. J Bone Joint Surg 1973; 55A : 535-44.

8 Marmor L. Textbook of Arthritis Surgery. Philadelphia: Lea and Febiger, 1976.

9 Arthritis and Rheumatism Council and British Orthopaedic Association. Controlled trial of synovectomy of knee and metacarpophalangeal joints in rheumatoid arthritis. Ann Rheum Dis 1976; 35: 437-42.

10 Arthritis Foundation Committee on Evaluation of Synovectomy. Multicenter evaluation of synovectomy in the treatment of rheumatoid arthritis. Report of results at the end of three years. Arthritis Rheum 1977; 20: 765-71.

11 Aidem H P, Baker L D. Synovectomy of the knee joint in rheumatoid arthritis. JAMA 1964; 187: 104-6.

12 Stevens J, Whitefield G A. Synovectomy of the knee in rheumatoid arthritis. Ann Rheum Dis 1966; 25: 214-9.

13 Paradies L H. Synovectomy of the knee. In early synovectomy in rheumatoid arthritis. In: Hijmans W, Paul
W D, Hershel H, eds. Early Synovectomy in Rheumatoid Arthritis. Amsterdam: Excepta Medica, 1969: 129-45.

14 Taylor A R, Harbison J S, Pepler C. Synovectomy of the knee in rheumatoid arthritis. 2. Results of surgery. Ann Rheum Dis 1972; 31: 159-61.

15 Graham J, Checketts R G. Synovectomy of the knee in rheumatoid arthritis. J Bone Joint Surg 1973; 55B: 786-95.

16 Gariepy R, Demers R, Laurin C A. The prophylactic effect of synovectomy of the knee in rheumatoid arthritis. Can Med Assoc J 1967; 94: 1349-52.

17 Fujikawa K, Yokoi A, Tanaka Y, Tomatsu T, Iseki F. Indication of early synovectomy of rheumatoid knee from view point of synovial proliferation by arthrographic study. J Rheum Arthritis Joint Surg (Jpn) 1978; 6: 51-9.

18 Geens S, Clayton M L, Leidholt J D. Synovectomy and Debridement of the knee in rheumatoid arthritis. Part 2. Clinical and roentgenographic study of thirty-one cases. J Bone Joint Surg 1969; 51A: 626-42.

19 Taylor A R, Hill A G S. Synovectomy. Clin Rheum Dis $1978 ; 4: 287-309$.

20 Ranawat C S, Desai K. Role of early synovectomy of the knee joint in rheumatoid arthritis. Arthritis Rheum 1975; 18: 117-21.

21 McEvan C. Early synovectomy in the treatment of rheumatoid arthritis. $N$ Engl J Med 1968; 279: 420-5.

22 Michell N S, Shepard N. The effect of synovectomy on synovium and cartilage in early rheumatoid arthritis. Orthop Clin North Am 1973; 4: 1057-84.

23 Kodama T, Kondo Y. Problems in early synovectomy. The fine structures of the synovial vessels in the knee joint. Clin Orthop Surg (Jpn) 1973; 8: 100-8. 